\section{Group-Individual POLYLIN}

DAVID J. WEISS

California State University, Los Angeles, California 90032

and

\section{JAMES C. SHANTEAU \\ Kansas State University, Manhattan, Kansas 66502}

POLYLIN (Shanteau, 1977) is a FORTRAN program for conducting analysis of variance tests on multiplicative trend components of the interactions in a factorial design. Such tests are used to evaluate multiplying models in a functional measurement framework (Anderson, 1982). Group-Individual POLYLIN is an extension that allows a group analysis when several subjects have judged the same set of stimuli. The extension supplants the technically incorrect procedure proposed for group designs by Graesser and Anderson (1974) and recommended with the earlier POLYLIN.

The program employs a least squares procedure that maximizes the sum of squares in the multilinear (e.g., linear-by-linear) component of each interaction. This is accomplished by using the marginal means for each factor as a basis for constructing its orthogonal trend coefficients following the algorithm presented by Weiss (1980). Coefficients are computed separately for each subject, and then a standard repeated-measures analysis (Keppel, 1973,pp. 414-419) is conducted.

Programmed in BASIC.PLUS for an interactive system, Group-Individual POLYLIN is entirely conversational. Data may be input from the terminal during execution or read from a file. Error correction after the data have been entered is available. Optional outputs include cell means, marginal means, and trend coefficients for each subject. The trend tests may be con- ducted for some or all of the components individually or for the multilinear component and pooled remainder of each interaction.

Limitations. As programmed for a PDP-11/70, Group-Individual POLYLIN comprises more than 700 lines. Factorial designs with from two to four factors (not including subjects or replicates) may be analyzed. Dimensioned for eight subjects with up to 400 scores/subject (these parameters may be adjusted via DIM statements), it occupies sufficient space to require chaining into two subprograms and considerable use of virtual arrays. Virtual storage slows execution a good deal, and users with larger machines may not need it. System-dependent features of BASIC-PLUS have been avoided and only two-dimensional arrays are used, so any machine of comparable size (the 11/70 allows $16 \mathrm{~KB}$ for a program) should be able to accommodate the program.

Availability. A listing of the program may be obtained without charge from David J. Weiss, Department of Psychology, California State University, Los Angeles, California 90032.

\section{REFERENCES}

ANDERson, N. H. Methods of information integration theory. New York: Academic Press, 1982.

Graesser, C. C., \& Anderson, N. H. Cognitive algebra of the equation: Gift size $=$ generosity $\times$ income. Journal of Experimental Psychology, 1974, 103, 692-699.

KEPPEL, G. Design and analysis: $A$ researcher's handbook. Englewood Cliffs, N.J: Prentice-Hall, 1973.

Shanteau, J. C. POLYLIN: A FORTRAN IV program for the analysis of multiplicative (multilinear) trend components of interactions. Behavior Research Methods \& Instrumentation, 1977, 9, 381-382.

WEIss, D. J. ORPOCO: Orthogonal polynomial coefficients. Behavior Research Methods \& Instrumentation, 1980, 12, 635.

(Accepted for publication June 1, 1982.) 